\title{
LA INVESTIGACIÓN ACCIÓN COMO ESTRATEGIA DE REVISIÓN DE LA PRÁCTICA PEDAGÓGICA EN LA FORMACIÓN INICIAL DE PROFESORES DE EDUCACIÓN BÁSICA
}

\section{A PESQUISA-AÇÃO COMO ESTRATÉGIA DE REVISÃO DA PRÁTICA PEDAGÓGICA NA FORMAÇÃO INICIAL DE PROFESSORES DA EDUCAÇÃO BÁSICA}

\author{
ACTION RESEARCH AS A STRATEGY FOR REVIEWING PEDAGOGICAL \\ PRACTICE IN THE INITIAL EDUCATION OF BASIC EDUCATION TEACHERS
}

Juan Pablo Catalán CUETO ${ }^{1}$

RESUMEN: El objetivo de esta investigación fue describir los fundamentos teóricos hacia una aproximación de aplicación de la investigación acción reflexión en el hacer docente durante las prácticas educacionales (2003-2004) por los estudiantes de pedagogía en Educación Básica de una Universidad Privada de Santiago. Las bases teóricas estudiadas fueron las de Argyris y Schón (1996) con la teoría de la acción, Elliot (1998) con investigación acción y Tonucci (1999) investigación en el aula, entre otros autores. La metodología inicial fue la investigación documental y luego aplicada como una aproximación a la investigación acción en el contexto escolar. El resultado fue un proceso satisfactorio de formación en los estudiantes y docentes en ejercicio apoyado en la investigación educativa, con una planificación, acción y reflexión en la búsqueda del cambio desde la escuela.

PALABRAS CLAVES: Investigación Acción. Formación Docente. Docente crítico reflexivo.

RESUMO: O objetivo desta pesquisa foi descrever os fundamentos teóricos para uma abordagem da aplicação da pesquisa-reflexão-ação no ensino durante as práticas educacionais (2003-2004) por alunos de pedagogia da Educação Básica de uma Universidade Privada de Santiago. As bases teóricas estudadas foram as de Argyris e Schón (1996) com a teoria da ação, Elliot (1998) com a pesquisa-ação e Tonucci (1999) com a pesquisa em sala de aula, entre outros autores. A metodologia inicial foi a pesquisa documental e posteriormente aplicada como abordagem à pesquisa-ação em contexto escolar. $O$ resultado foi um processo de formação satisfatório para alunos e professores em exercício, amparado na pesquisa educacional, com planejamento, ação e reflexão na busca pela mudança da escola.

PALAVRAS-CHAVE: Pesquisa-ação. Formação docente. Docente crítico-reflexivo.

ABSTRACT: The aim of this paper was to describe the theoretical foundations towards an approach of application of the research action reflection in the teaching practice (2003-2004) by the students of pedagogy in Basic Education of a Private University of Santiago. The theoretical bases studied were those of Argyris and Schon (1996) with the theory of action, Elliot (1998) with action research and Tonucci (1999) research in the classroom, among other

${ }^{1}$ Universidad SEK (USEK), Santiago - Chile. Coordinador de la Unidad de Currículum y Evaluación de la Dirección de Docencia. ORCID: https://orcid.org/0000-0003-4702-8839. E-mail: juanpablo.catalan@usek.cl

RIAEE - Revista Ibero-Americana de Estudos em Educação, Araraquara, v. 15, n. esp. 4, p. 2768-2776, dez., 2020. e-ISSN: 1982-5587 
authors. The initial methodology was documentary research and then applied as an approach to action research in the school context. The result was a satisfactory process of training in students and teachers in practice supported by educational research, with planning, action and reflection in the search for change from the school.

KEYWORDS: Action research. Teacher education. Critically-reflective teacher.

\section{Introducción}

Los rápidos cambios sociales y tecnológicos exigen la construcción de nuevas imágenes de la práctica social en general y de las empresas en particular. Se piensa que la indagación realizada por los entes de estas instituciones puede constituirse en una de las herramientas de cambio y mejora en la calidad organizacional. En el ámbito educativo, cualquier profesional de la docencia vinculado al mundo de las instituciones, está llamado a desempeñar un papel clave como investigador de su propia práctica con la finalidad de mejorar su formación, su desempeño en el aula, escuela y comunidad en la búsqueda de un cambio organizacional hacia una transformación sociocultural.

Esta investigación presenta algunos de los fundamentos teóricos con una aproximación a la aplicación de la investigación acción en el hacer docente durante las prácticas educacionales de los docentes de Pedagogía de Educación Básica que fue desarrollada en las Escuelas Básicas de la Región Metropolitana de Chile.

En el trabajo se defiende: Que la investigación debe ser el eje central colaborativo de las organizaciones. El papel de los participantes, pasantes y profesores de las de instituciones universitarias como alianzas estratégicas, se puede considerar como promotores pedagógicos investigadores sobre la práctica para el mejoramiento de la escuela. Que la investigación debe realizarse desde y para las organizaciones. La investigación tiene sentido en el entorno de los problemas escolares. La investigación desde y para la escuela se constituye en una excelente herramienta para mejorar la calidad de las organizaciones.

\section{Desarrollo}

La investigación como actividad humana es la función más trascendental de la sociedad. No se puede proceder a la deriva, es necesario conocer los hechos, causas relaciones y consecuencias en toda la fase del proceso, esto debe hacerse en plena conciencia de todos sus elementos y factores si se desea lograr la eficacia. La investigación debe despertar la curiosidad, la reflexión, el cuestionamiento, la duda, bases fundamentales de toda genuina investigación. 
De allí la investigación será educativa si permite que los participantes involucrados desarrollen nuevas formas de comprensión y si le forman para emprender caminos propios de reflexión autónoma y compartida sobre el sentido de la práctica y las posibilidades de mejorarla. En el contexto educativo puede considerarse como un encuentro entre personas, es una actividad ética que requiere de continua reflexión y cuestionamiento, para ello no puede reducirse a una actividad técnica, debido a la profundidad del proceso, en ella participan docente-alumno comunidad, en la cual, el docente actúa con todo lo que él es como sujeto, es decir, su comportamiento está enmarcado en sus creencias, actitudes, costumbres y entorno.

De igual manera el alumno recibe información y la procesa de acuerdo con sus experiencias, costumbres y entorno, por ello el proceso de aprendizaje es una situación incierta, única, cambiante, compleja y presenta conflicto de valores tanto en la definición de las metas como en la selección de los medios. Al docente le toca intervenir en un medio escolar comunitario complejo, en un escenario psico-social-espiritual vivo y cambiante, definido por la interacción simultanea de múltiples factores (sociales, económicos, culturales, políticos, entre otros) y condiciones.

El éxito consiste en la habilidad para manejar la complejidad y resolver problemas prácticos, que más que problemas son situaciones problemáticas. Esto supone un proceso de reflexión en la acción o una conversación reflexiva con la situación problemática concreta que permitirá crear nuevas realidades, corregir e inventar. Se trata de actuar de forma inteligente y creativa o de poner en acción las ideas que el considere pertinente después de su reflexión ante los conflictos. La reflexión en la acción, (que se realiza mientras se actúa) la reflexión sobre la acción (análisis posterior a la acción) e incluso la reflexión sobre la reflexión en la acción (somete a un cuestionamiento más profundo) la propia reflexión es el mejor instrumento para un aprendizaje significativo y para un genuino desarrollo profesional y para iniciar el camino hacia el cambio.

Cuando el docente investiga en y sobre su acción, se convierte en un investigador en el aula en su escuela y comunidad. No depende ya ni de técnicas, rutina, recetas, normas ni de prescripciones curriculares impuestas desde afuera por los "expertos" los programas y los textos. Depende fundamentalmente de sus propios descubrimientos, de la teoría que va elaborando en su hacer pedagógico reflexionado permanentemente. El maestro construye su propia teoría, la aplica y verá su propio resultado positivo o negativo, reflexiona, corrige y reconstruye de nuevo. El genuino investigador busca la comprensión de fenómenos y procesos, más que la acumulación de datos, asume el método dialéctico de investigación- reflexiónacción, en la cual partiendo de la experiencia la somete a cuestionamiento y la reelabora. Por 
esto se puede afirmar, que la función principal de la investigación educativa consiste en sensibilizar, lograr que todos sean conscientes de los problemas.

Una rigurosa actividad investigadora en y sobre el hacer docente y el aprendizaje, agudiza la reflexión, orienta la atención hacia los aspectos importantes, aclarar los problemas, estimular el debate y el intercambio de opiniones, profundizando así el entendimiento la flexibilidad y la adaptación, aspirando incrementar la capacidad en resolución de problemas. Toda investigación supone la incesante búsqueda de la verdad, el desentrañar lo aparente, la precisión, el rigor y objetividad en el conocimiento la exploración, creatividad imaginación, duda constante, actitud crítica; en la formulación permanente de porqué, búsqueda de explicaciones para todas las cosas, autodisciplina, perseverancia y el trabajo metódico en un ambiente que fomente: curiosidad, búsqueda, experimentación y modos naturales de aprender.

Por esto, es urgente que el docente logre estimular en los alumnos la curiosidad de saber, preguntar, explorar, comprobar, experimentar, perfeccionar, aprender por deseo, no por miedo u obligación. Fomentar en ellos el sano hábito de dudar, enseñarlos a construir, formular y expresar con libertad sus preguntas. ayudarles a razonar, comprender, argumentar, defender su punto de vista, aceptar y respetar posturas diferentes a ver "la cosa" desde diversos ángulos. Se debe combatir la memorización mecánica a favor de un aprendizaje significativo, basado en la comprensión, razonamiento, explicación y descripción de los hechos.

Crear la necesidad de la lectura comprensiva y crítica, y la escritura sistemática. Desarrollar el gusto y la capacidad de observación agudizando los sentidos registrando cuidadosamente lo observado. Privilegiar los métodos de aprendizaje que permiten aprender haciendo, comprobando y experimentado. Estimular en el alumno: el ser exigente consigo mismo, a no darse por vencido, a intentar hasta lograrlo, a ser seguro con lo que dice y hace. Se puede asegurar, que solo el docente capaz de vivir él mismo la experiencia de investigar de forma auténtica, podrá promocionar y garantizar una labor de investigación correcta en los alumnos. El adulto que quiere contagiar a los niños actitudes de investigación como la necesidad de: discutir, profundizar hallar soluciones, buscar nuevos caminos, poner en práctica conocimientos procesados, ha de ser un adulto que vive (en su vida personal) estas actitudes (TONUCCI, 1999) las cuales forman parte epistemológica de su práctica (reflexión permanente y simultanea de su acción educativa) cuya dinámica del proceso permite conocer la realidad y aprender de lo nuevo, porque no hay fórmulas preestablecidas.

Se hace necesario que el docente tenga formación sobre los tipos de investigación, aunque el más ajustado a la realidad y proceso de aprendizaje en el aula y escuela es el de investigación acción, aunado a la reflexión sobre su práctica. 


\section{La Investigación Educativa}

Es una indagación sistemática y autocrítica, basada en la curiosidad estable y en el deseo de comprensión de una situación problemática. Es una tarea cotidiana, una herramienta y un pasatiempo, su utilidad incide en las mentes de los hombres o seres involucrados. La investigación educativa genera una transformación en el hacer docente, debido a que busca el trabajo en equipo, cooperativo, comunitario con una secuencia sistemática y con cambios permanentes en el proceso, con el objetivo de llegar al contexto a la realidad social de los actores.

La investigación y la evaluación sobre currículo y el aprendizaje que se realice en la escuela y sus aulas, es moldeado por medio de un paradigma. Este paradigma se inclina por la acción transformadora de cambiar la educación y tener un proceso educativo que concuerde con el hecho social, encontrando así la perspectiva necesaria para llegar de forma más clara al objetivo a lograr, esa forma es la investigación para y en el aula.

En la investigación educativa se desarrolla desde el proceso de evaluación que parte a el diagnostico, y que presentan las siguientes características: Se analizan las acciones humanas de padres y alumnos situaciones sociales comunitarios experimentadas por los docentes en la escuela. Se relaciona con los problemas del día a día que en lo cotidianos se confrontan para la búsqueda de una solución al problema. La educación necesita atención, que solvente los problemas de improvisación y facilismo que utilizan los maestros para impartir los contenidos de la forma más cómoda y rutinaria, teniendo de esta forma el punto de partida necesario para el comienzo de una nueva forma de educar a través de la investigación como herramienta fundamental de las docentes, dándoles la función y el deber ser investigadores e compartir estos conocimientos e implementar un método innovador que de mejores resultados en las aulas de clases (GONZÁLEZ, 2005)

\section{La Investigación en el Aula}

Distintas propuestas didácticas han aparecido en los últimos años con la finalidad de encontrar una solución viable que aporte una esperanza para el sistema educativo del país. Se ofrece la oportunidad de aplicar la investigación como estrategia adecuada para la construcción de sus propios conocimientos, fomentando sus conceptos, sus actitudes y sus intereses con un mecanismo que genere motivación en los alumnos, debido a que son ellos los personajes principales y los más interesados en lograr un aprendizaje óptimo, pertinente y necesario. 
En esta propuesta se ofrece un docente que aborde la investigación como una tarea que solvente sus inquietudes de planificación, estrategias y prácticas educativas, surgiendo de ser un sujeto que se lleva por lo cotidiano y pasivo a ser completamente activo que maneje la educación cooperativamente en conjunto con la comunidad y los alumnos, con el fin colectivo de tener un sistema educativo mucho mejor. Notándose de otra forma, la propuesta parte del conocimiento cotidiano y habitual en la solución de problemas que conlleva al alumno a un conocimiento científico y una percepción más amplia sobre determinado contenido.

Con esta propuesta se enfoca la investigación científica como el método para solventar problemas escolares cotidianos y que tanto el docente como los alumnos manejen este procedimiento, de llegar al objetivo común para todos los actores o involucrados que será siempre el de lograr el cambio en la práctica pedagógica del sistema escolar. La investigación para los docentes a iniciar la IA desde el aula.

\section{Estrategias Investigativas de Aula}

Según Tonucci (1999), la investigación surge de la necesidad de un cambio frente a problemas existentes en el sistema educativo desde el aula, la solución se enfoca en utilizar los mismos conocimientos del currículo antes utilizados en los planes y programas, pero de forma nueva y creativa, tomándolo como método y estrategia para realizar la labor de educar.

El autor trata de explicar que se busca en todo tiempo que los conocimientos afloren para resolver problemas cotidianos que en cualquiera momento estén al alcance del alumno y de ninguna manera exigir soluciones a problemas que no entiendan y que no le sean pertinentes, tampoco se busca que los alumnos sean abarrotados de contenidos teóricos para ser sometidos a un régimen de preguntas y respuestas que solo ponen a prueba la capacidad memorística de los alumnos, sin absorber un aprendizaje significativo.

El docente puede ingeniarse muchas formas de inducir a los alumnos desde su aula a investigar sin seguir una receta establecida que sea igual para todos, utilizando instrumentos o herramientas como:

- Libros, periódicos y revistas

- Encuestas que indaguen sobre el tema a investigar desde el hogar y comunidad

- Entrevistas con objetivos claros y precisos

- Fotos, diapositivas, álbumes

- Recolección de elementos relacionados con la investigación

RIAEE - Revista Ibero-Americana de Estudos em Educação, Araraquara, v. 15, n. esp. 4, p. 2768-2776, dez., 2020. e-ISSN: 1982-5587 
- La Internet, entre otros

Es necesario que el docente se dedique a la función de investigar con sus alumnos en el hacer pedagógico, tomándolos en cuenta en decidir el tema a investigar y tener como punto de partida sus intereses, indicar a los niños solo el propósito objetivos a lograr y que ellos mismos sean creadores de un procedimiento que ayude a su aprendizaje y que lejos de ser una investigación confiable y científica, sea un trabajo que ayude a descubrir aspectos nuevos de la realidad de su contexto e inspire a estos niños a seguir investigado y que sea el comienzo de un cambio educativo desde el aula, utilizando el PPA como estrategia investigativa.

El maestro debe orientar y mediar en su labor a los niños a la investigación permanente y delimitar la misma para evitar la dispersión del tema central, orientando en todo momento las actividades que el mismo debe crear y proponer, pero no decidir, eso lo harán los propios alumnos. Se pueden nombrar algunas estrategias y métodos para aplicar la investigación en el aula, es indispensable situarse en un determinado nivel educativo y con alumnos de una determinada edad debido a que las estrategias tienen un nivel de exigencia y dificultad y deben ser adaptadas a las edades de los estudiantes.

En los proyectos pedagógicos de aula (PPA), se manejan todas estas características ya planteadas debido a que está basado en un aprendizaje cooperativo y con la investigación inmersa como estrategia o como eje transversal.

\section{Resultados y conclusiones}

Un ejemplo de investigación acción en las escuelas como centros de aplicación de las prácticas profesionales. La investigación se centró en la metodología de investigación acción reflexión, desarrollada en fases y momentos, los cuales se desarrollaron en dos escuelas.

Luego de haber sistematizado las bases teóricas de varias fuentes bibliográficas y aplicado el método de investigación acción reflexión con el equipo de pasantes promotores en las Escuelas como centros de aplicación de las prácticas profesionales de los profesores de educación básica.

La investigación y la reflexión en la acción docente, representa una nueva forma de la práctica, lo que lleva a replantear la función de los profesionales de la docencia, a un cambio profundo en su hacer y en el proceso de formación y desarrollo humano. 
La Investigación acción requiere que los investigadores trabajen en equipo y se comprometan a efectuar innovaciones, y que crean en la posibilidad de llegar al cambio desde una perspectiva positiva y democrática desde el aula hacia la escuela y la comunidad.

Es emergente estimular en los alumnos la curiosidad de saber, preguntar, explorar, comprobar, experimentar, perfeccionar, aprender por deseo, no por presión u obligación como tareas a cumplir. Se debe fomentar en ellos el sano hábito de dudar, enseñarlos a construir, formular y expresar con espontaneidad, libertad en sus preguntas, ayudarles a razonar, comprender, argumentar, defender su punto de vista, aceptar y respetar la diversidad y posiciones diferentes, a ver desde diversos ángulos. Evitar la memorización mecánica a favor de un aprendizaje constructivo significativo, basado en la comprensión, razonamiento, explicación y descripción.

Es necesario que antes de comenzar cualquier proceso de investigación en la escuela o en el aula, los alumnos estén claros en que la intención no es solucionar el problema sino buscar un mecanismo didáctico que solvente las inquietudes e intereses de ellos sobre determinado problema o contenido. Para solucionar es necesario un procedimiento netamente metodológico que no todos los alumnos tienen la madurez para su comprensión.

La finalidad de la investigación en el hacer docente es partir desde y para el alumno con métodos, técnicas y estrategias, donde ellos participen, decidan y sean personajes, fomentando el ser y el hacer para un conocer y convivir. Los maestros son los responsables del hacer en las aulas de clase, este puede considerarse un laboratorio de investigación educativa, los maestros son los que deben comprobar día a día las diferentes teorías educativas y construir las propias, mediante la observación deben descubrir las potencialidades del alumno, ambiente, de la escuela y la comunidad.

Los maestros generalmente expresan que no cuentan con tiempo disponible para la investigación, sin considerar que dentro del aula es una acción natural y tiene las herramientas más preciadas como son las experiencias de los alumnos en la ejecución de los proyectos pedagógicos de aula, entre otras actividades que se desarrollan diariamente en el contexto escolar para desarrollar el proyecto de la escuela integrado a la comunidad. Los estudiantes en proceso de formación deben desarrollar competencias investigativas desde el centro de estudio y ejercitarlo en el centro de aplicación, con ello podrá resolver cualquier conflicto cognitivo, procedimental o actitudinal en su hacer como futuro docente. 


\section{REFERENCIAS}

ARGYRIS, C; SCHÓN, D. Organizational learning II: theory, method, and practice.

Reading, MA: Addison Wesicy, 1996.

ELLIOT, J. La investigación acción en educación. 2. ed. Morata, 1998.

ESTÉ, A. El Aula Punitiva. Caracas, Venezuela: Trópicos, 1998

FRIEDMAN, V. Action Science: Creating communities of inquirí incommunities of practice. In: REASON, P.; BRADBURY, H. Handbook of action research. London: Sage, 2001. p. 159-170.

GONZÁLEZ, N. Trabajo de ascenso de la categoría profesor titular LUZ. 2005.

GREENWOOD, D; LEVIN, M. Introduction to Action Research. Social Research for Social Change. London: Sage, 1998

KEMMIS, S. Teoría crítica de la enseñanza. La investigación acción en la formación de I profesorado. Barcelona: Martínez Roca, 1988.

KEMMIS, S.; MCTAGGART, T. Cómo planificar la investigación acción. Barcelona: Laertes, 1988.

STRINGER, E. T. Action Research. A Handbook for Practitioners. London. Sage Publications. In: Tercer Encuentro Iberoamericano de Colectivos Escolares y Redes de maestros que hacen investigación en la Escuela. ¿Cómo circulan y se producen los saberes y las artes en la escuela? 2002.

TONUCCI, F. La Investigación como alternativa a la enseñanza. ¿Enseñar Aprender? Editorial Laboratorio Educativo. 2. ed. Caracas, Venezuela, 1999.

\section{Cómo referenciar este artículo}

CUETO, J. P. C. La investigación acción como estrategia de revisión de la práctica pedagógica en la formación inicial de profesores de educación básica. Revista Ibero-Americana de Estudos em Educação, Araraquara, v. 15, n. esp. 4, p. 2768-2776, dez., 2020. e-ISSN: 19825587. DOI: https://doi.org/10.21723/riaee.v15iesp4.14534

Remitido el: 10/09/2019

Revisiones requeridas el: 10/01/2020

Aprobado el: 30/04/2020

Publicado el: 01/12/2020 